\title{
Oleic acid triggers hippocampal neurogenesis by binding to TLX/NR2E1
}

Prasanna Kandel ${ }^{1,2,} \uparrow$, Fatih Semerci ${ }^{3,4} \uparrow$, Aleksandar Bajic ${ }^{4,5}$, Dodge Baluya ${ }^{6}, \mathrm{LiHua} \mathrm{Ma}^{7}$, Kevin Chen ${ }^{8}$, Austin $\mathrm{Cao}^{9}$, Tipwarin Phongmekhin ${ }^{8}$, Nick Matinyan ${ }^{1,10}$, William Choi ${ }^{4,11}$, Alba Jiménez-Panizo ${ }^{12}$, Srinivas Chamakuri ${ }^{2}$, Idris O. Raji ${ }^{2}$, Lyra Chang ${ }^{2}$, Pablo Fuentes-Prior ${ }^{13}$, Kevin R. MacKenzie $2,7,14$, Caroline L. Benn ${ }^{15}$, Eva Estébanez-Perpiñáa ${ }^{12}$, Koen Venken ${ }^{1,10}$, David D. Moore ${ }^{1,16}$, Damian W.Young ${ }^{1,2,4,9,14,17, *}$, and Mirjana Maletic-Savatic ${ }^{3,4,16,17,18^{*}}$

Affiliations: ${ }^{1}$ Integrative Molecular and Biomedical Sciences, Graduate Program Baylor College of Medicine, Houston, TX, 77030, USA

${ }^{2}$ Department of Pharmacology \& Chemical Biology, Baylor College of Medicine, One Baylor Plaza, Houston, Texas 77030, USA.

${ }^{3}$ Department of Pediatrics, Baylor College of Medicine, Baylor College of Medicine, Houston, TX, 77030, USA.

${ }_{4}^{4} J a n$ and Dan Duncan Neurological Research Institute, Texas Children's Hospital, Houston, TX, 77030, USA.

${ }^{5}$ Department of Molecular and Human Genetics, Baylor College of Medicine, Houston, TX, 77030, USA.

${ }^{6}$ Department of Interventional Radiology, University of Texas, MD Anderson, Houston, TX, 77030, USA.

${ }^{7}$ NMR and Drug Metabolism Advanced Technology Cores, Baylor College of Medicine, Houston, TX, 77030, USA.

${ }^{8}$ Department of Biosciences, Rice University, Houston, TX, 77005, USA.

${ }^{9}$ Department of Chemistry, Rice University, Houston, TX 77005, USA.

${ }^{10}$ Verna and Marrs McLean Department of Biochemistry and Molecular Biology, Baylor College of Medicine, Houston, TX, 77030, USA.

${ }^{11}$ Medical Scientist Training Program, Baylor College of Medicine, Houston, TX, 77030, USA.

${ }^{12}$ Department of Biochemistry and Molecular Biomedicine, Institute of Biomedicine (IBUB), University of Barcelona (UB), 08028 Barcelona, Spain.

${ }^{13}$ Molecular Basis of Disease, Biomedical Research Institute Sant Pau (IIB Sant Pau), 08025 Barcelona, Spain.

${ }^{14}$ Department of Pathology and Immunology, Baylor College of Medicine, One Baylor Plaza, Houston, Texas 77030, USA.

${ }^{15}$ Pfizer Regenerative Medicine, The Portway, Granta Park, Cambridge CB21 6GP.

${ }^{16}$ Department of Molecular and Cellular Biology, Baylor College of Medicine, Houston, TX, 77030, USA.

${ }^{17}$ Center for Drug Discovery, Baylor College of Medicine, Houston, TX, 77030, USA.

${ }^{18}$ Department of Neuroscience, Baylor College of Medicine, Houston, TX, 77030, USA.

$\uparrow$ These authors contributed equally to this work

* Correspondence to: Damian.Young@bcm.edu and Mirjana.Maletic-Savatic@bcm.edu

Current affiliations:

William Choi: Department of Obstetrics and Gynecology, Tulane University, 1430 Tulane Avenue \#8611, New Orleans, LA 70112 
Dodge Baluya: Tissue Imaging, Metabolomics and Proteomics Laboratory, Washington State University, Pullman, WA 99163

Idris O. Raji: Koch Institute, MIT, 500 Main St., Cambridge, MA 02142

Kevin Chen: The Pritzker School of Medicine, University of Chicago, 924 E. 57th Street, Chicago, IL 60637

Austin Cao: Perelman School of Medicine, University of Pennsylvania, 3400 Civic Center Blvd, Philadelphia, PA, 19104

Caroline Benn: CSO LoQus23 Therapeutics, Riverside, Babraham Research Campus, Cambridge, CB22 3AT, United Kingdom 


\begin{abstract}
Adult hippocampal neurogenesis underpins learning, memory, and mood, but diminishes with age and illness. The orphan nuclear receptor TLX/NR2E1 is known to regulate neural stem and progenitor cell self-renewal and proliferation, but the precise mechanism by which it accomplishes this is unknown. We found that neural stem and progenitor cells require monounsaturated fatty acids to survive and proliferate. Specifically, oleic acid (18:1 $\omega 9)$ binds to TLX to convert it from a transcriptional repressor to a transcriptional activator of cell cycle and neurogenesis genes. We propose a model in which sufficient quantities of this endogenous ligand must bind to TLX to trigger the switch to proliferation. These findings pave the way for future therapeutic manipulations to counteract pathogenic impairments of neurogenesis.
\end{abstract}


The mammalian brain contains a population of neural stem cells that continue to produce functional neurons well into adulthood (1-4). This ongoing capacity to form new neurons, known as adult neurogenesis, enables learning and memory $(5,6)$ and supports proper mood regulation (7). Conversely, impairments in neural stem cell proliferative capacity and neuronal commitment are associated with advanced age $(8,9)$ and disorders such as depression $(10)$ and Alzheimer's disease $(11,12)$. Indeed, antidepressant therapies such as serotonin reuptake inhibitors are effective only insofar as they succeed in stimulating neurogenesis (7). There has thus been considerable interest in learning how to manipulate hippocampal neurogenesis in order to treat a range of conditions.

One key question is how to preserve the pool of neural stem and progenitor cells while promoting the ongoing generation of neurogenic progeny. To maintain their population, these cells must be able to self-renew, and it is well established that neural stem and progenitor cell self-renewal and proliferation are regulated by the transcription factor TLX (also known as NR2E1) (13-15). Unfortunately, TLX is an orphan nuclear receptor-in other words, no endogenous ligand for TLX has yet been discovered (exogenous and synthetic ligands have been reported but without physiological relevance in vivo $(16,17)$ ). Without such a ligand, TLX cannot be validated as a therapeutic target for promoting neurogenesis.

We are not without clues as to the probable nature of such a ligand, however. First, nuclear receptors as a class tend to bind to lipophilic molecules, such as hormones, vitamins, and fatty acids. Second, neural stem cells require de novo lipogenesis to proliferate (18-20). Third, we previously identified a nuclear magnetic resonance (NMR) signal that correlates with neurogenic activity in mice and humans; it resonates at $1.28 \mathrm{ppm}$, a frequency that corresponds to the saturated hydrocarbon groups that characterize all fatty acids (21). Fourth, blocking the activity of fatty acid synthase, which catalyzes the synthesis of saturated fatty acids, markedly reduces the $1.28 \mathrm{ppm}$ NMR signal (21) and prevents running from stimulating neurogenesis in mice (18). We therefore hypothesized that fatty acids would be crucial to TLX function.

In fact, compared to other cells such as astrocytes and fully differentiated neurons, neural stem cells have particularly high levels of mono-unsaturated fatty acids (MUFAs) (21). Interestingly, while most of the metabolic genes enriched in quiescent neural stem cells are associated with fatty acid synthesis, these cells have particularly high expression of stearoyl-CoA desaturases $(22,23)$, which are involved specifically in the synthesis of MUFAs. This suggests that MUFAs might have a special role in controlling the cell cycle in neural stem cells. In this study, we asked whether MUFA synthesis is necessary for survival and proliferation of these cells, and whether these fatty acids might act as ligands for TLX in neural stem cells.

\section{MUFAs are essential for neural stem and progenitor cell survival and proliferation}

To begin to address these questions, we first confirmed that MUFAs are abundant in human neural stem and progenitor cells. Two-dimensional ${ }^{1} \mathrm{H}-{ }^{1} \mathrm{H}$ COSY NMR, which identifies coupled hydrogen $\left({ }^{1} \mathrm{H}\right)$ spins, revealed the mono-unsaturated bond of MUFAs in these cells (fig. S1A). Next, gas chromatography-mass spectrometry found several saturated fatty acid precursors and MUFAs, with oleic acid (18:1 $\omega 9$ ) being the most abundant (fig. S1B). Like their murine counterparts, human neural stem and progenitor cells require fatty acid synthase to survive: 
cerulenin, an inhibitor of fatty acid synthase, decreased their viability in a dose-dependent manner (fig. S2A). Treating the cells with CAY10566, an inhibitor of the stearoyl-CoA desaturases that catalyze the conversion of saturated fatty acids into MUFAs, resulted in a doseand time-dependent impairment of survival in vitro (Fig. 1A, left panel). Strikingly, this impairment could be rescued by exogenous 18:1 MUFA but not 18:0 saturated fatty acids (Fig. 1A, right panel; fig. S2B). Consistent with these in vitro data, oral administration of CAY10566 in mice significantly reduced neural stem and progenitor cell proliferation in vivo (Fig. 1B). Therefore, human neural stem and progenitor cells require de novo MUFA synthesis for proliferation and survival.

If MUFA synthesis is required for survival of these cells, then MUFAs should be abundant in the dentate gyrus of the hippocampus, where neurogenesis occurs. Using imaging mass spectrometry to map lipids in the mouse brain, we detected both 18:0 saturated fatty acids $(\mathrm{m} / \mathrm{z}=283.24)$ and 18:1 MUFA $(\mathrm{m} / \mathrm{z}=281.24)$ in the dentate gyrus. In order to gauge the concentration, we spiked $0.5 \mathrm{mM}$ 18:1 19 control onto the thalamus (Fig. 1C, fig. S3). The 18:1 MUFA ion showed a very similar concentration in the dentate gyrus, where it was most abundant. We therefore considered the possibility that the MUFAs are not being used just for energy metabolism, lipid membrane formation, and so forth, but could also serve as signaling molecules that regulate neurogenesis.

\section{Oleic acid binds to the TLX ligand-binding domain}

Because there is no crystal structure of TLX bound to ligands, we searched for a nuclear receptor that binds fatty acids and whose ligand-binding domain has an established structure. The closest liganded homolog is HNF4 $\alpha$ (fig. S4). Initial structural studies revealed HNF4 $\alpha$ bound to fatty acids $(24,25)$ and functional studies indicate that HNF4 $\alpha$ is selectively activated by exogenous

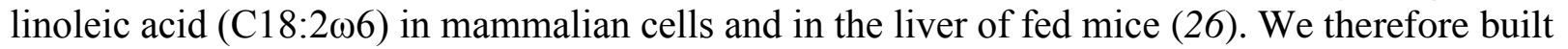
a homology model of the human TLX ligand-binding domain and performed molecular docking based on the X-ray crystal structure of fatty acid-bound HNF4 $\alpha$ ligand-binding domain (PDB $1 \mathrm{M} 7 \mathrm{~W})$. These docking studies suggested that fatty acids could indeed fit into the binding pocket and potentially function as cognate ligands of TLX (fig. S4E).

To determine whether fatty acids actually bind to the purified TLX ligand-binding domain (fig. S5A), we used biolayer interferometry to compare the binding responses of different fatty acids with an18 carbon chain length (Fig. 2A). Stearic acid (18:0 saturated fatty acid) had no observable binding, while $\alpha$-linolenic acid (18:3 $\omega 3$ poly-unsaturated fatty acid) failed to reach equilibrium or saturate over the concentrations tested, suggesting a weak binding interaction. Oleic acid (18:1 199 MUFA), however, displayed a saturable binding response with a $K_{D}$ of 7.3 $\mu \mathrm{M}$. Interestingly, the synthetic 18:1 $\omega 5$ MUFA had a similar saturable binding response with a $\mathrm{K}_{\mathrm{D}}$ of $6.5 \mu \mathrm{M}$ (fig. S5B). These experiments show that MUFAs, such as 18:1 $\omega 9$, can bind to the TLX ligand-binding domain.

\section{Oleic acid binding to TLX promotes recruitment of co-activators}

To understand the functional effects of MUFA binding to TLX, we considered that in its basal state, TLX recruits corepressor proteins such as atrophins (ATN 1 and 2) (27), lysine-specific demethylase (LSD1) (28), and histone deacetylases (HDAC 1, 3, and 5) to potently repress 
transcription (29). We first confirmed the direct interaction between TLX and the atrophin corepressor peptide using biolayer interferometry and found that the atrophin peptide binds TLX with a $\mathrm{K}_{\mathrm{D}}$ of $14.2 \mu \mathrm{M}$ (fig. S6A). We next used the Amplified Luminescent Proximity Homogeneous Assay (ALPHA) to measure the interaction of atrophin peptide and TLX ligandbinding domain. Competition with untagged atrophin peptide attenuated the ALPHA signal with an $\mathrm{EC}_{50}$ of $1.3 \mu \mathrm{M}$, demonstrating that the assay can detect both TLX-corepressor binding and its disruption (fig. S6B). To determine which fatty acids could disrupt TLX-atrophin peptide interaction, we examined a panel of fatty acids (saturated, mono-unsaturated, and polyunsaturated) (Fig. 2B). The saturated fatty acids (14:0, 16:0 and 18:0) had no effect on the ALPHA signal, indicating that they do not interfere with the interaction of atrophin with the TLX

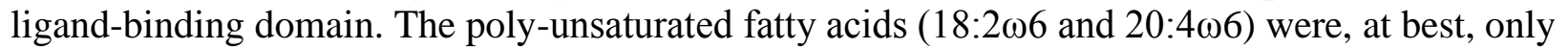

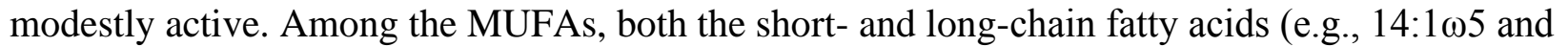

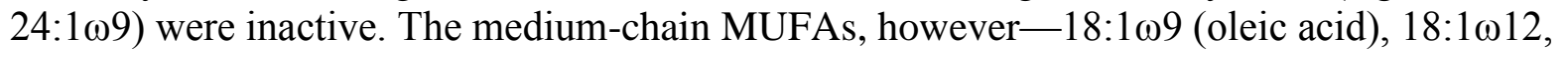
and 20:1 19 - clearly disrupted the interaction of TLX with the corepressor. Out of the entire panel, cis 18:1 19 (oleic acid) reduced the interaction with atrophin the most. Given that it was also the most abundant MUFA in human neural stem and progenitor cells (fig. S1B) and the most potent fatty acid in the panel at a single dose, we next performed dose-response

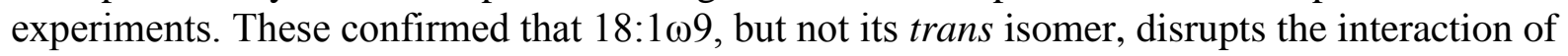
the TLX ligand-binding domain with the atrophin peptide in a dose-dependent manner, with an $\mathrm{EC}_{50}=19.3 \mu \mathrm{M}$ (Fig. 2C). We conclude that the TLX ligand-binding domain stereospecifically recognizes certain fatty acids and that occupancy of the TLX ligand-binding pocket by specific MUFAs, especially oleic acid, elicits conformational changes that disengage the atrophin corepressor.

Could fatty acid ligands induce TLX to switch from corepressor to coactivator binding? TLX interacts with nuclear receptor coactivators such as NCOA1, NCOA2, NCOA3 (SRC1-3) (30). To examine whether oleic acid-bound TLX could recruit NCOA1, we again used the ALPHA screen, placing a tagged NCOA1-II coactivator peptide containing the canonical LXXLL nuclear receptor interaction motif and the TLX ligand-binding domain on donor and acceptor beads, respectively. In the absence of fatty acids or in the presence of only saturated or poly-unsaturated fatty acids, there was no interaction between the NCOA1-II peptide and the TLX ligand-binding domain, but 18:1 19 markedly increased the ALPHA signal (Fig. 2D). As with disruption of the TLX-corepressor interaction, enhancement of the TLX-coactivator interaction was specific to

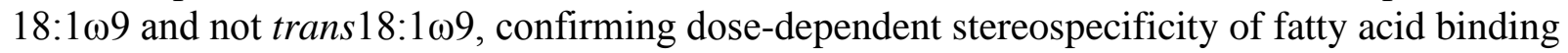
and coactivator recruitment (Fig. 2E). To determine the affinity of coactivator binding, we performed competition experiments and found that, in the presence of saturating 18:1 $\omega 9$, TLX recruited both the NCOA1-II peptide and a receptor-interaction domain from NCOA3, with $\mathrm{EC}_{50}$ values of $6.0 \mu \mathrm{M}$ (fig. S6C) and $3.3 \mu \mathrm{M}$ (fig. S6D), respectively. A TLX-based Homogeneous Time-Resolved Fluorescence assay independently verified ligand-dependent coactivator recruitment and the specificity of cis/trans 18:1 $\omega 9$ recognition (Fig. 2F). These data strongly indicate that oleic acid binding determines whether TLX functions as a transcriptional repressor or an activator.

To examine the effect of 18:1 199 on TLX-mediated transcription, we built a dual luciferase transcription reporter system with a previously reported TLX-binding response element (31). We cotransfected TLX with the reporter plasmids in HeLa cells grown in media with charcoal- 
stripped fetal bovine serum to minimize fatty acids and other non-polar molecules. Luciferase expression was reduced in these cells compared to cotransfection with the empty TLX expression vector (Fig. 3A, left panel), confirming the repressive function of TLX at baseline. The predicted agonist activity of 18:1 109 was confirmed by the expected dose-dependent increase in luciferase expression (Fig. 3A, middle panel). This effect was not seen with trans18:169 (Fig. $3 \mathrm{~A}$, right panel; fig. S6E), verifying that the modest and stereospecific recognition of the cisform was retained in living cells and that 18:1 $\omega 9$ binding promotes TLX-mediated transcriptional activation of TLX-dependent genes.

\section{Oleic acid-activated TLX triggers neural stem cell mitosis and neurogenesis in vivo}

To test the effect of 18:1 199 on adult neurogenesis in vivo, we stereotactically delivered either $18: 1 \omega 9,18: 3 \omega 3$ (as a fatty acid control), or sham to the left dentate gyrus, then treated mice with BrdU analogs to label newborn cells, and sacrificed them at different timepoints post-injection (Fig. 3B-D; Tables S1, S2). Compared to controls, $24 \mathrm{hr}$ after injection the percentage of proliferating $\mathrm{EdU}^{+}$neural stem cells and their progeny (amplifying neuroprogenitors) was elevated at the site of 18:109 injection only (Fig. 3B, C; Table S1A). The stimulatory effect of $18: 1 \omega 9$ is thus specific and local. The number of immature $\left(\mathrm{Dcx}^{+}, \mathrm{CldU}^{+}\right)$and mature $\left(\mathrm{NeuN}^{+}\right.$, $\mathrm{CldU}^{+}$) newborn neurons rose 30 days following 18:1 $\omega 9$ injection (Fig. 3D; Table S2), confirming neurogenesis had been stimulated. The percentage of proliferating $\left(\mathrm{IdU}^{+}\right)$neural stem cells and amplifying neuroprogenitors at this timepoint did not, however, differ from controls, indicating that the stimulatory effect of exogenous 18:1 199 on these cells is short-lived (fig. S7).

To determine whether 18:1 $\omega 9$ requires TLX to affect neural stem cell function, we took advantage of $L f n g$-eGFP transgenic mice, in which eGFP selectively labels radial neural stem cells (32). We crossed Tl $x^{\operatorname{loxp} / \text { loxp }}$ mice (13) with Lfng-eGFP; $L f n g-C r e E R^{\mathrm{T} 2}$;RCL-tdT mice to achieve homozygous deletion of $T l x$ in Cre-induced neural stem cell following tamoxifen administration (fig. S8). In the resulting iTl $x^{\mathrm{fl} / \mathrm{fl}}$ mice, wild-type neural stem cells (no Cre activation) have two copies of $T l x\left(T l x^{+++}\right)$and are labeled green (eGFP). Mutant neural stem cells (Cre-induced) lack both copies of $T l x\left(T l x^{\mathrm{fl} / \mathrm{fl}}\right)$ and are labeled both red (tdT) and green (eGFP). Thus, in iTlx ${ }^{\mathrm{fl} / f \mathrm{l}}$ brains we can examine 18:1 $\omega 9$-dependent cell-autonomous effects in both wild-type and mutant neural stem cells from the same dentate gyrus. Following a single 18:1 199 injection, the ratio of proliferating neural stem cells in $\mathrm{i} T l x^{\mathrm{fl} / \mathrm{fl}}$ mice correlated with $T l x$ gene dosage: the percentage of EdU ${ }^{+}$cells was much higher when two copies of $T l x$ were present than when only one copy was present, and 18:1 199 exerted no proliferative effect when both copies of Tlx were lacking (Fig. 3E; Table S1B). Thus, independent of any purely metabolic role for MUFA, the stimulatory effects of 18:1 19 on adult hippocampal neural stem cells and neurogenesis are mediated through TLX.

Our next step was to determine whether 18:1 $\omega 9$-activated neural stem cell proliferation

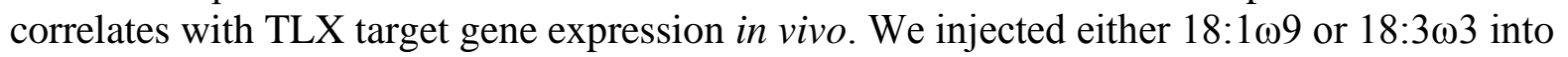
both dentate gyri of $L f n g$-eGFP mice and performed RT-PCR on sorted neural stem cells to measure expression of known TLX targets as well as a panel of cell cycle and neurogenesis genes. To qualify as being activated in a 18:1 $\omega 9$-dependent manner, a given gene should be

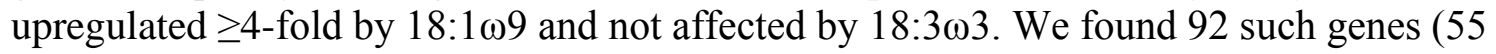
involved in the cell cycle and 37 in neurogenesis) (Fig. 4A). (An additional 11 cell cycle and 8 


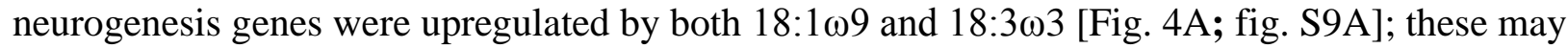
be nonspecific fatty acid responders and were excluded from further analyses.)

To determine which of the 92 18:1 19 -dependent genes are also TLX-dependent, we injected either 18:1 19 or sham into both dentate gyri of $\mathrm{i} T l x^{\mathrm{fl} / \mathrm{fl}}$ mice and performed RT-PCR on sorted neural stem cells again, using the same gene panels (Fig. 4B). We identified 48 cell cycle and 52 neurogenesis genes upregulated $\geq 4$-fold in neural stem cells lacking $T l x\left(T l x^{\mathrm{fl} / \mathrm{fl}} \mathrm{eGFP}^{+}, \mathrm{tdT}^{+}\right)$ compared to wild-type neural stem cells $\left(T l x^{+/+} \mathrm{eGFP}^{+}\right)$from sham-treated $i T l x^{\mathrm{fl} / \mathrm{fl}}$ mice. Among these genes, 45 cell cycle and 35 neurogenesis genes overlapped with those upregulated by

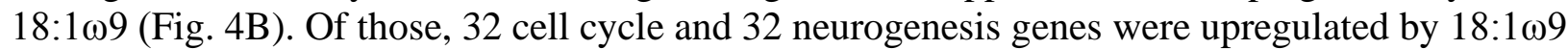
in a TLX-dependent manner (Fig. 4C). We did not pursue the basis of the response of the remaining 13/45 cell cycle and 3/35 neurogenesis genes that were not exclusively TLXdependent (fig. S9B, C), although they warrant further study.

The 18:1 $\omega 9$-activated TLX-dependent genes included previously reported TLX targets such as Ascll (13), Trp53 (33), Mcm2, and Ccnd2 (34), but also revealed new targets such as Olig2, Shh, Fgf2, Dvl3, Hey1, Pax6, Nf1, Dll1, Nr2e3,E2f2, E2f3, Cdk1, and Cdk2. It is worth noting that TLX does not act as a binary on-off switch: 18:1 $\omega 9$-bound TLX activates cell cycle genes more strongly than neurogenesis genes, while lack of TLX de-represses neurogenesis genes more strongly (Fig. 4C). This is consistent with previous reports that a gradual increase - and not a steady, high expression — of neurogenic genes, such as Ascll, is necessary for neuroprogenitors to slowly enter into cell cycle to avoid premature differentiation (35-37).

\section{Discussion}

This study thus sheds new light on the importance of fatty acid metabolism and monounsaturated fatty acids as signaling molecules. Oleic acid is not only essential for neural stem cell proliferation and neurogenesis in the hippocampus, but it appears to help preserve the neural stem cell population over time. Because TLX activation requires relatively high levels of oleic acid, neural stem cells are not perpetually dividing and quickly depleting in number. Our results support a model in which oleic acid acts as a critical signaling metabolite in neural stem cells. In its ligand-free state, TLX favors corepressor binding and repression of its target genes, causing these cells to remain quiescent. During quiescence, oleic acid is synthesized and accumulates until it reaches a certain threshold that indicates the availability of metabolic prerequisites for cell cycle entry. Upon binding to oleic acid, TLX disengages from the corepressors and recruits transcriptional coactivators, which triggers neural stem cell proliferation. In response to acutely increased oleic acid levels, robust upregulation of cell cycle genes would prompt neural stem cells to generate neurogenic progeny while preventing their direct differentiation into neurons. Temporally cued de-repression of neurogenesis genes could then ensure that the progeny properly proceed into the neurogenic and not astrocytic lineage.

A recent study reported that TLX is also a receptor for exogenous natural and synthetic retinoids but did not explore the physiology of these interactions in vivo (16). Here, by demonstrating that oleic acid binding to TLX is crucial for stimulating neurogenesis, this work lays a foundation for future efforts to preserve neural stem cells during aging and in disease. The discovery of an endogenous ligand for TLX will facilitate efforts to identify small molecules that can modulate TLX function and perhaps eventually lead to cell-based repair and neural regeneration. 
Acknowledgments: We thank C. Benod and P. Webb for assistance in early work; the late A. Sarrion-Perdigones for assistance with the dual luciferase reporter assay; R. Evans for sharing the $T l x^{\mathrm{fl} / \mathrm{fl}}$ mice; $\mathrm{P}$. Yi for providing plasmids to express GST-NCOA3-RID; the BCM Center for Drug Discovery; the BCM NMR and Drug Metabolism Advanced Technology Core; M. Wang and J. Wang for access to GC-MS instrumentation, and C.-C. Lin and S.L. Holmes for technical support on GC-MS; J. Dannison and the MD Anderson Cancer Center Imaging Mass Spectrometry core for instruments and technical support. We thank Bert O'Malley, H. Zoghbi, M. Matzuk, P. Lucassen, and members of the Maletic-Savatic and Young laboratories for comments on the manuscript and helpful discussions; and V. Brandt for expert editing and comments on the work.

Funding: This project was supported by the Cytometry and Cell Sorting Core at BCM with funding from the CPRIT Core Facility Support Award (CPRIT-RP180672), the NIH (CA125123 and RR024574) and the assistance of J.M. Sederstrom; and the BCM IDDRC Grant (P50HD10355) from the Eunice Kennedy Shriver National Institute of Child Health and Human Development for use of the Microscopy Core facilities, the RNA In Situ Hybridization Core facility, and the Human Neuronal Differentiation Core facility. The work was partially supported by Baylor College of Medicine (BCM) start-up funds, the Albert and Margaret Alkek Foundation, the McNair Medical Institute at The Robert and Janice McNair Foundation, the Cancer Prevention and Research Institute of Texas (CPRIT) grant R1313 (V.K.); the R. P. Doherty, Jr. Welch Chair in Science (Q-0022, D.D.M.), BCM Seed Funding 1P20CA22173101A1 (D.W.Y.); and NIGMS R01 GM120033, Cynthia and Antony Petrello Endowment, Mark A. Wallace Endowment, McKnight Foundation, Dana Foundation, and BCM Computational and Integrative Biomedical Research Center seed grant (M.M-S.). The authors acknowledge Diana Helis Henry Medical Research Foundation and the Adrienne Helis Malvin Medical Research Foundation through its direct engagement in medical research in conjunction with BCM and the [Oleic acid promotes adult hippocampal neurogenesis by acting as an endogenous ligand for nuclear receptor TLX] program.

Author Contributions: P.K. and F.S. contributed to experimental design, performed in vitro and in vivo experiments, respectively, analyzed the data, and drafted the manuscript. A.B. supplied human neural stem and progenitor cells and assisted in their analyses. D.B. and K.C. contributed to the IMS data acquisition and analyses. L.M. and K.R.M. performed the NMR experiments and analyses. A.C. and T.P. conducted luciferase and ALPHA screen assays. N.M. cloned the luciferase system. W.C. contributed first binding data. A.J-P., P.F-P., C.L.B. and E. E-P. contributed tools and data interpretation. S.C. synthesized fatty acid. I.O.R. conducted docking experiments. L.C. cloned and optimized the in vitro methods. K.V. supervised the luciferase experiments. D.D.M. contributed to the overall concepts, experimental design, and data interpretation. D.W.Y. and M.M-S. conceived and designed the experiments, oversaw the overall execution of the project, interpreted data, provided financial support, and wrote the manuscript. All authors discussed the results and commented on manuscript.

Competing interests: the authors declare no competing interests.

Correspondence and requests for materials should be addressed to M. Maletic-Savatic (Mirjana.Maletic-Savatic@bcm.edu) or D.W. Young (Damian.Young@bcm.edu). 
bioRxiv preprint doi: https://doi.org/10.1101/2020.10.28.359810; this version posted October 29, 2020. The copyright holder for this preprint (which was not certified by peer review) is the author/funder. All rights reserved. No reuse allowed without permission. 


\section{References:}

1. J. Altman, Are new neurons formed in the brains of adult mammals? Science 135, 1127 1128 (1962).

2. H. G. Kuhn, H. Dickinson-Anson, F. H. Gage, Neurogenesis in the dentate gyrus of the adult rat: age-related decrease of neuronal progenitor proliferation. J. Neurosci. 16, 20272033 (1996).

3. K. L. Spalding et al., Dynamics of hippocampal neurogenesis in adult humans. Cell 153, 1219-1227 (2013).

4. M. Boldrini et al., Human Hippocampal Neurogenesis Persists throughout Aging. Cell Stem Cell 22, 589-599 e585 (2018).

5. C. D. Clelland et al., A functional role for adult hippocampal neurogenesis in spatial pattern separation. Science 325, 210-213 (2009).

6. N. S. Burghardt, E. H. Park, R. Hen, A. A. Fenton, Adult-born hippocampal neurons promote cognitive flexibility in mice. Hippocampus 22, 1795-1808 (2012).

7. A. S. Hill, A. Sahay, R. Hen, Increasing Adult Hippocampal Neurogenesis is Sufficient to Reduce Anxiety and Depression-Like Behaviors. Neuropsychopharmacology 40, 23682378 (2015).

8. J. M. Encinas et al., Division-coupled astrocytic differentiation and age-related depletion of neural stem cells in the adult hippocampus. Cell Stem Cell 8, 566-579 (2011).

9. N. Urban et al., Return to quiescence of mouse neural stem cells by degradation of a proactivation protein. Science 353, 292-295 (2016).

10. B. A. Samuels, R. Hen, Neurogenesis and affective disorders. Eur. J. Neurosci. 33, 11521159 (2011).

11. E. P. Moreno-Jimenez et al., Adult hippocampal neurogenesis is abundant in neurologically healthy subjects and drops sharply in patients with Alzheimer's disease. Nat. Med. 25, 554-560 (2019).

12. M. K. Tobin et al., Human Hippocampal Neurogenesis Persists in Aged Adults and Alzheimer's Disease Patients. Cell Stem Cell 24, 974-982 e973 (2019).

13. C. L. Zhang, Y. Zou, W. He, F. H. Gage, R. M. Evans, A role for adult TLX-positive neural stem cells in learning and behaviour. Nature 451, 1004-1007 (2008).

14. K. Murai et al., Nuclear receptor TLX stimulates hippocampal neurogenesis and enhances learning and memory in a transgenic mouse model. Proc. Natl. Acad. Sci. U. S. A. 111, 9115-9120 (2014).

15. Y. Shi et al., Expression and function of orphan nuclear receptor TLX in adult neural stem cells. Nature 427, 78-83 (2004).

16. K. Griffett et al., The Orphan Nuclear Receptor TLX Is a Receptor for Synthetic and Natural Retinoids. Cell Chem Biol, (2020).

17. C. Benod et al., The human orphan nuclear receptor tailless (TLX, NR2E1) is druggable. PLoS One 9, e99440 (2014).

18. N. E. Chorna et al., Fatty acid synthase as a factor required for exercise-induced cognitive enhancement and dentate gyrus cellular proliferation. PLoS One 8, e 77845 (2013).

19. M. Knobloch et al., Metabolic control of adult neural stem cell activity by Fasndependent lipogenesis. Nature 493, 226-230 (2013). 
20. M. Bowers et al., FASN-Dependent Lipid Metabolism Links Neurogenic Stem/Progenitor Cell Activity to Learning and Memory Deficits. Cell Stem Cell 27, 98109 e111 (2020).

21. L. N. Manganas et al., Magnetic resonance spectroscopy identifies neural progenitor cells in the live human brain. Science 318, 980-985 (2007).

22. J. Shin et al., Single-Cell RNA-Seq with Waterfall Reveals Molecular Cascades underlying Adult Neurogenesis. Cell Stem Cell 17, 360-372 (2015).

23. P. Codega et al., Prospective identification and purification of quiescent adult neural stem cells from their in vivo niche. Neuron 82, 545-559 (2014).

24. G. B. Wisely et al., Hepatocyte nuclear factor 4 is a transcription factor that constitutively binds fatty acids. Structure 10, 1225-1234 (2002).

25. K. Duda, Y. I. Chi, S. E. Shoelson, Structural basis for HNF-4alpha activation by ligand and coactivator binding. J. Biol. Chem. 279, 23311-23316 (2004).

26. X. Yuan et al., Identification of an endogenous ligand bound to a native orphan nuclear receptor. PLoS One 4, e5609 (2009).

27. C. L. Zhang, Y. Zou, R. T. Yu, F. H. Gage, R. M. Evans, Nuclear receptor TLX prevents retinal dystrophy and recruits the corepressor atrophin1. Genes Dev. 20, 1308-1320 (2006).

28. A. Yokoyama, S. Takezawa, R. Schule, H. Kitagawa, S. Kato, Transrepressive function of TLX requires the histone demethylase LSD1. Mol. Cell. Biol. 28, 3995-4003 (2008).

29. G. Sun, R. T. Yu, R. M. Evans, Y. Shi, Orphan nuclear receptor TLX recruits histone deacetylases to repress transcription and regulate neural stem cell proliferation. Proc. Natl. Acad. Sci. U. S. A. 104, 15282-15287 (2007).

30. X. Corso-Diaz et al., Co-activator candidate interactions for orphan nuclear receptor NR2E1. BMC Genomics 17, 832 (2016).

31. D. Wu et al., Orphan nuclear receptor TLX functions as a potent suppressor of oncogeneinduced senescence in prostate cancer via its transcriptional co-regulation of the CDKN1A (p21(WAF1) (/) (CIP1) ) and SIRT1 genes. J. Pathol. 236, 103-115 (2015).

32. F. Semerci et al., Lunatic fringe-mediated Notch signaling regulates adult hippocampal neural stem cell maintenance. Elife 6, (2017).

33. H. K. Liu et al., The nuclear receptor tailless induces long-term neural stem cell expansion and brain tumor initiation. Genes Dev. 24, 683-695 (2010).

34. W. Niu, Y. Zou, C. Shen, C. L. Zhang, Activation of postnatal neural stem cells requires nuclear receptor TLX. J. Neurosci. 31, 13816-13828 (2011).

35. J. Andersen et al., A transcriptional mechanism integrating inputs from extracellular signals to activate hippocampal stem cells. Neuron 83, 1085-1097 (2014).

36. A. Raposo et al., Ascl1 Coordinately Regulates Gene Expression and the Chromatin Landscape during Neurogenesis. Cell Rep 10, 1544-1556 (2015).

37. S. Lugert et al., Homeostatic neurogenesis in the adult hippocampus does not involve amplification of Ascl1(high) intermediate progenitors. Nat Commun 3, 670 (2012). 


\section{Figure Legends}

Fig. 1 Human neural stem and progenitor cells depend on de novo mono-unsaturated fatty acid (MUFA) synthesis to survive and proliferate. (A) Acetyl coenzyme A (AcetylCoA) and methyl-malonyl CoA serve as substrates for saturated fatty acid (SFA) synthesis catalyzed by fatty acid synthase (FASN). SFAs are converted to MUFAs via desaturases, such as the stearoylCoA desaturases (SCD), which catalyze the rate-limiting step in the MUFA synthesis. Left panel: Dose-dependent human neural stem and progenitor cell viability following SCD inhibition with CAY10566 (SCDi), normalized to vehicle (DMSO) response. Right panel: Dosedependent human neural stem and progenitor cell viability following $144 \mathrm{hr}$ exposure to SCDi and then treated with 18:1 19 MUFA or its precursor, 18:0 SFA, normalized to the respective treatment ( $\mathrm{N} \geq 3$ per group). Statistics was done using two-way ANOVA and Tukey's multiple comparisons test, $* * * * \mathrm{p} \leq 0.0001$. (B) $\mathrm{SCDi}(3 \mathrm{mg} / \mathrm{kg}$ body weight $)$ or sham $(0.03 \mathrm{~N} \mathrm{HCl})$ was delivered orally to 2-3-month-old wild-type C57BL/6J mice followed by EdU $(50 \mathrm{mg} / \mathrm{kg}$ body weight) to label proliferating human neural stem and progenitor cells in vivo ( $\mathrm{N}=3$ per group). Bar graphs represent mean $\pm \mathrm{SEM}$. ${ }^{*} \mathrm{p} \leq 0.05$. Representative confocal micrographs show proliferating $\mathrm{Edu}^{+} \mathrm{GFAP}^{+} \mathrm{Sox} 2^{+}$human neural stem and progenitor cells. Scale bar $=100 \mu \mathrm{m}$. (C) Imaging mass spectrometry of the 3-month-old wild-type C57BL/6J mouse brain shows distribution of the 18:0 SFAs (m/z 283.24, left panel), the precursor of 18:1 MUFAs (m/z 281.24, right panel). Dentate gyrus (DG) and CA1 region of hippocampus are indicated (arrows). See also figs. S1-3.

Fig. 2 18:1 19 MUFA binds to TLX ligand-binding domain, reduces the corepressor and enhances the coactivator interaction. (A) Dose-dependent binding response of different classes of fatty acids with the same carbon chain length (18 carbons) to the TLX ligand-binding domain, using biolayer interferometry label-free technology. 18:0 SFA has no observable binding response (left panel). 18:1 19 MUFA binding response fit is a saturable one-site fit (the dissociation constant $\left(\mathrm{K}_{\mathrm{d}}\right)=7.3 \mu \mathrm{M}, 95 \% \mathrm{CI}=0.385-14.2 \mu \mathrm{M}$; middle panel $)$. 18:3 03 polyunsaturated fatty acid (PUFA) binding response fit is non-saturable $\left(\mathrm{K}_{\mathrm{d}}=270.9 \mu \mathrm{M}, 95 \%\right.$ $\mathrm{CI}=140.8-400.9 \mu \mathrm{M}$, right panel). (B) Normalized Amplified Luminescent Proximity Homogeneous Assay (ALPHA) screen for the interaction between TLX ligand-binding domain and a corepressor (atrophin peptide) in the presence of selected MUFAs, PUFAs, and SFAs at 25 $\mu$ M. Signal $<1.0$ indicates decreased interaction. (C) Dose-dependent TLX ligand-binding domain/corepressor interaction in the presence of 18:1 $\omega 9\left(\mathrm{EC}_{50}=19.3 \mu \mathrm{M}, 95 \% \mathrm{CI}=18.7-19.5\right.$ $\mu \mathrm{M}$ ) or trans 18:1 199 MUFA (synthetic stereoisomer). (D) Normalized ALPHA screen of the interaction between TLX ligand-binding domain and a coactivator (NCOA1 peptide) in the presence of selected MUFAs, PUFAs, and SFAs screened at $50 \mu \mathrm{M}$. Signal $>1.0$ indicates increased interaction. Note the interaction in the presence of 18:1 109 versus trans $18: 1 \omega 9$. (E) Dose-dependent TLX ligand-binding domain/coactivator interaction in the presence of 18:1 $\omega 9$ $\left(\mathrm{EC}_{50}=59.8 \mu \mathrm{M}, 95 \% \mathrm{CI}=50.6-70.8 \mu \mathrm{M}\right)$ or trans 18:1 $\omega 9$. (F) Dose-dependent Homogeneous Time Resolved Fluorescence (HTRF) signal for TLX/coactivator peptide recruitment in the

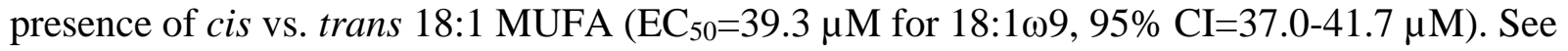
also figs. S4-6.

Fig. 3 18:109-dependent TLX effect on murine adult neural stem cell proliferation and neurogenesis in vivo. (A) TLX-based dual luciferase reporter activity in HeLa cells with or 
without TLX-expressing plasmids (left panel). Dose-dependent TLX-luciferase response in HeLa cells with or without TLX-expressing plasmids (middle panel). TLX-luciferase response in HeLa cells with TLX-expressing plasmids in the presence of cis or trans $18: 1 \omega 9$ (100 $\mu \mathrm{M}$ each) (right panel). Bar graphs represent mean \pm SEM. $* * * \mathrm{p} \leq 0.005, * * * * \mathrm{p} \leq 0.001$. (B) Representative confocal hippocampal sections show Lfng-eGFP $^{+}$neural stem cells (NSCs), Sox $2^{+}$amplifying neuroprogenitors (ANPs), and EdU ${ }^{+}$proliferating cells in the subgranular zone of a 2-month-old Lfng-eGFP mouse treated with either sham or 18:1 $\omega 9$. Scale bar $=100 \mu \mathrm{m}$. (C) The number of NSCs and ANPs in 2-month-old Lfng-eGFP mice injected with either sham (no solvent), 18:1 $\omega 9$, or 18:3 133 fatty acids into the left dentate gyrus (ipsilateral, IL) (300 nL of pure fatty acid per injection site); the right dentate gyrus (contralateral, CL) served as a non-injection control (N=35 per group). Sham served as an injection site control. 18:3 103 served as a fatty acid control. Bar graphs represent mean \pm SEM. For statistical analysis (two-way ANOVA, Tukey's multiple comparison test), CL and IL from the same mouse were compared (Table 1A). (D) The number of newborn immature neurons and granule cells in 2-month-old Lfng-eGFP mice treated

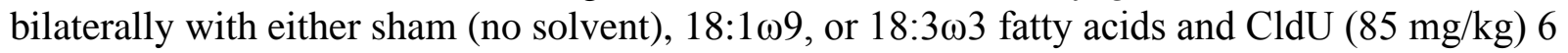
$\mathrm{hr}$ thereafter ( $\mathrm{N}=3-4$ per group). Bar graphs represent mean $\pm \mathrm{SEM}$; statistics was done using one-way ANOVA and Tukey's multiple comparison test (Table 2). Please see fig. S7 for quantification of IdU ${ }^{+} \mathrm{NSCs}$ and ANPs 1-month post-injection. (E) 2-month-old Lfng$\mathrm{CreER}^{\mathrm{T} 2} /$ Rosa26tdT/Lfng-eGFP/Tlx ${ }^{\mathrm{fl} / f \mathrm{l}}\left(\mathrm{i} T l x^{\mathrm{fl} / \mathrm{fl}}\right)$ mice were given tamoxifen (TMX; $120 \mathrm{mg} / \mathrm{kg}$ body weight) to induce CreER ${ }^{\mathrm{T} 2} 48 \mathrm{hr}$ before either sham (no solvent) or 18:1 $\omega 9$ delivery (300 $\mathrm{nL}$ of pure fatty acid per injection site), followed by EdU (50 mg/kg i.p.) $6 \mathrm{hr}$ and $22 \mathrm{hr}$ thereafter ( $\mathrm{N}=3$ per group). Confocal micrographs show EdU ${ }^{+}$proliferating cells in mice with two copies of $T l x\left(\mathrm{i} T l x^{+/+}\right)$, one copy of $T l x\left(\mathrm{i} T l x^{\mathrm{fl} /+}\right)$, and lacking both copies of $T l x\left(\mathrm{i} T l x^{\mathrm{fl} / \mathrm{fl}}\right)$. Bar

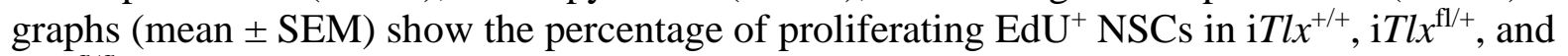
$\mathrm{i} T l x^{\mathrm{fl} / \mathrm{fl}}$ mice. For statistical analysis (two-way ANOVA, Tukey's multiple comparison test), CL and IL from the same mouse were compared (Table 1B). ${ }^{*} \mathrm{p} \leq 0.05, * * \mathrm{p} \leq 0.001, \mathrm{NS}=$ nonsignificant. See fig. S8 for generation of $i T l x^{\mathrm{fl} / \mathrm{fl}}$ mice.

Fig. 4 18:109 induces TLX to upregulate cell cycle and neurogenesis genes in adult murine hippocampal neural stem cells in a TLX-dependent manner. (A) 2-month-old Lfng-eGFP

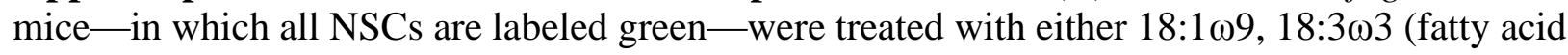
control), or sham ( $\mathrm{N}=3$ per group), and $\mathrm{eGFP}^{+} \mathrm{NSCs}$ were sorted from the dentate gyri. RT-PCR was done using a panel of cell cycle and neurogenesis genes. Venn diagram represents differentially regulated genes derived from pairwise $\Delta \mathrm{C}_{\mathrm{t}}$ value comparison across groups. Numbers in blue are cell cycle genes and numbers in red are neurogenesis genes. (B) 2-monthold iTlx ${ }^{\mathrm{fl} / \mathrm{fl}}\left(L f n g-\mathrm{CreER}{ }^{\mathrm{T} 2} / \mathrm{Rosa} 26 \mathrm{tdT} / L f n g-\mathrm{eGFP} / T l x^{\mathrm{fl} / \mathrm{fl}}\right)$ mice were given tamoxifen (TMX; 120 $\mathrm{mg} / \mathrm{kg}$ body weight) $48 \mathrm{hr}$ before either sham (no solvent) or 18:1 $\omega 9$ delivery ( $\mathrm{N}=3$ per group). $\mathrm{Tlx}^{\mathrm{fl} / \mathrm{fl}} \mathrm{NSCs}\left(\mathrm{tdT}^{+} \mathrm{eGFP}^{+}\right.$; orange) and $\mathrm{Tlx}^{+/+} \mathrm{NSCs}\left(\mathrm{eGFP}^{+}\right.$) were sorted from the dentate gyri. RT-PCR was done for the same cell cycle and neurogenesis genes as in Fig. 4A. Venn diagram represents differentially regulated genes derived from pairwise $\Delta \mathrm{C}_{\mathrm{t}}$ value comparison across groups. Numbers in blue are cell cycle genes and numbers in red are neurogenesis genes. (C) Heatmaps of $\Delta \Delta \mathrm{C}_{\mathrm{t}}$ values (cited in boxes) show 32 cell cycle and 32 neurogenesis genes exclusively upregulated by 18:1 $\omega 9$ ( $\geq 4$-fold change) in a TLX-dependent manner. 18:1 $\omega 9$ treated vs. sham-treated $T l x^{+/+}$NSCs $\left(L f n g\right.$-eGFP ${ }^{+}$NSCs from $L f n g$-eGFP mice) indicates genes

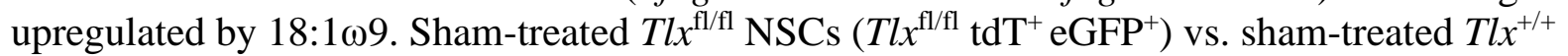
NSCs indicates genes de-repressed by TLX. See also fig. S9. 
FASN SCDs AcetylCoA + Malonyl-CoA $\longrightarrow$ SFA $\stackrel{\text { SCDS }}{\longrightarrow}$ MUFA
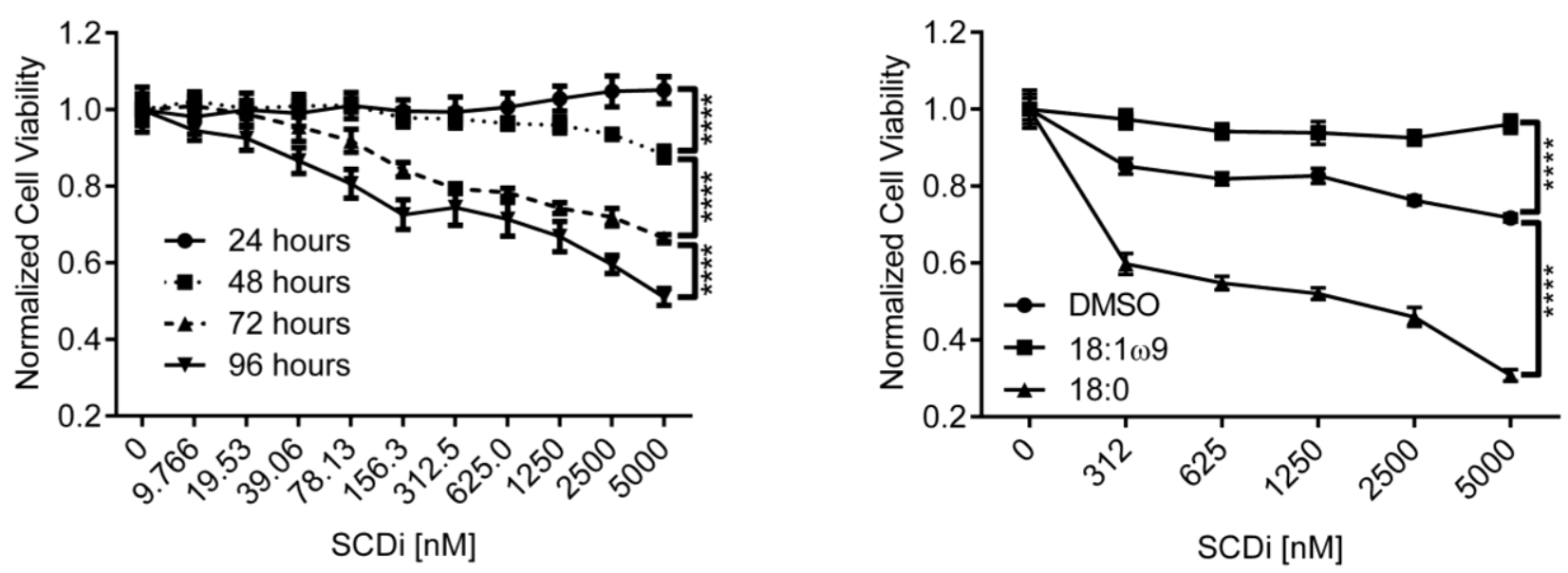

B
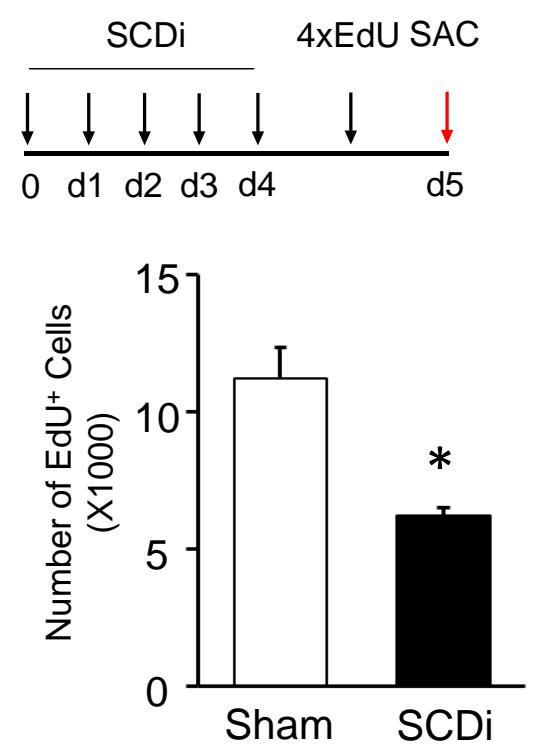

Control

SCDi

\section{8:0 (m/z 283.24)}

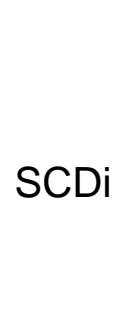

C
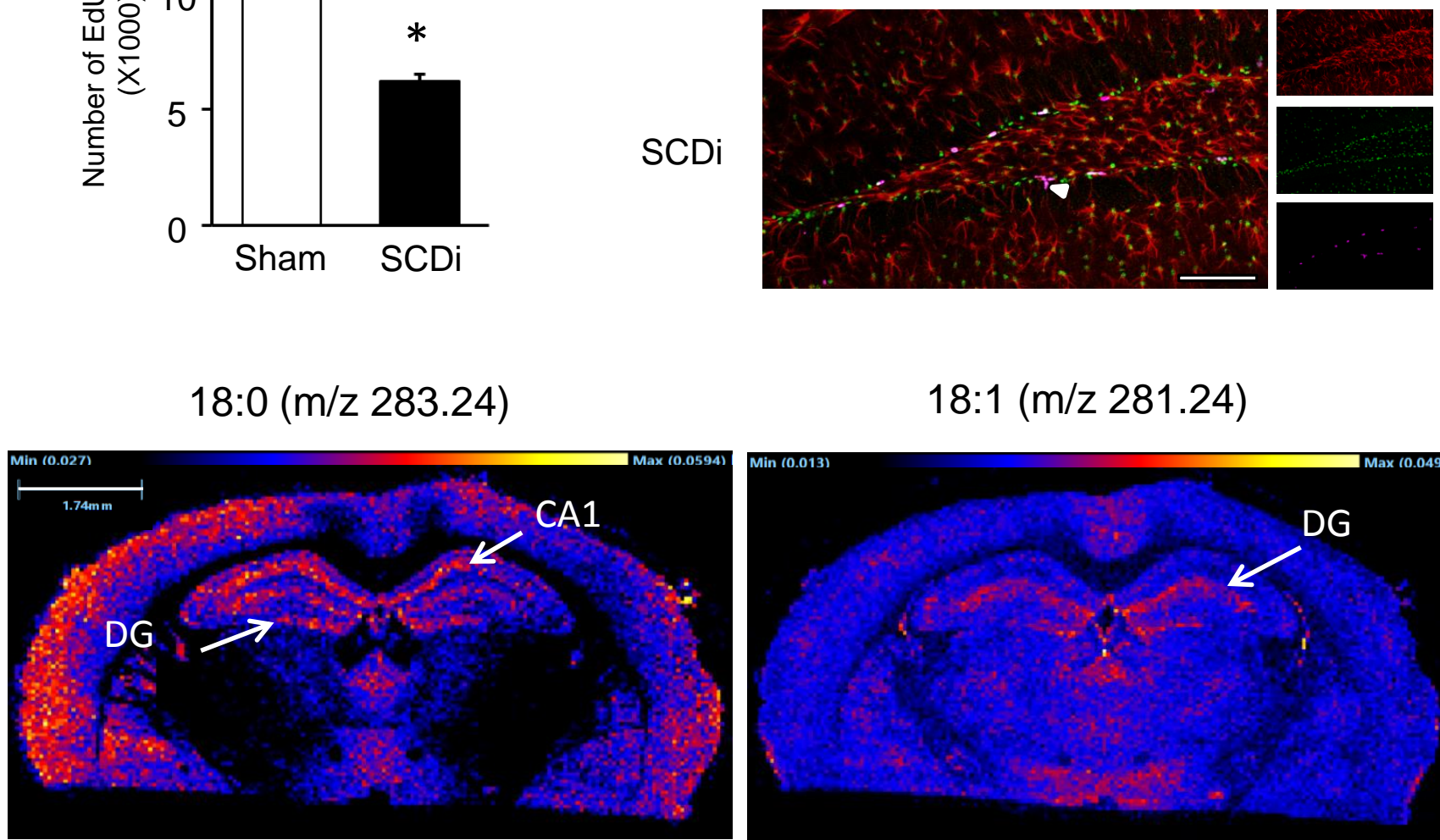

$18: 1(\mathrm{~m} / \mathrm{z} 281.24)$
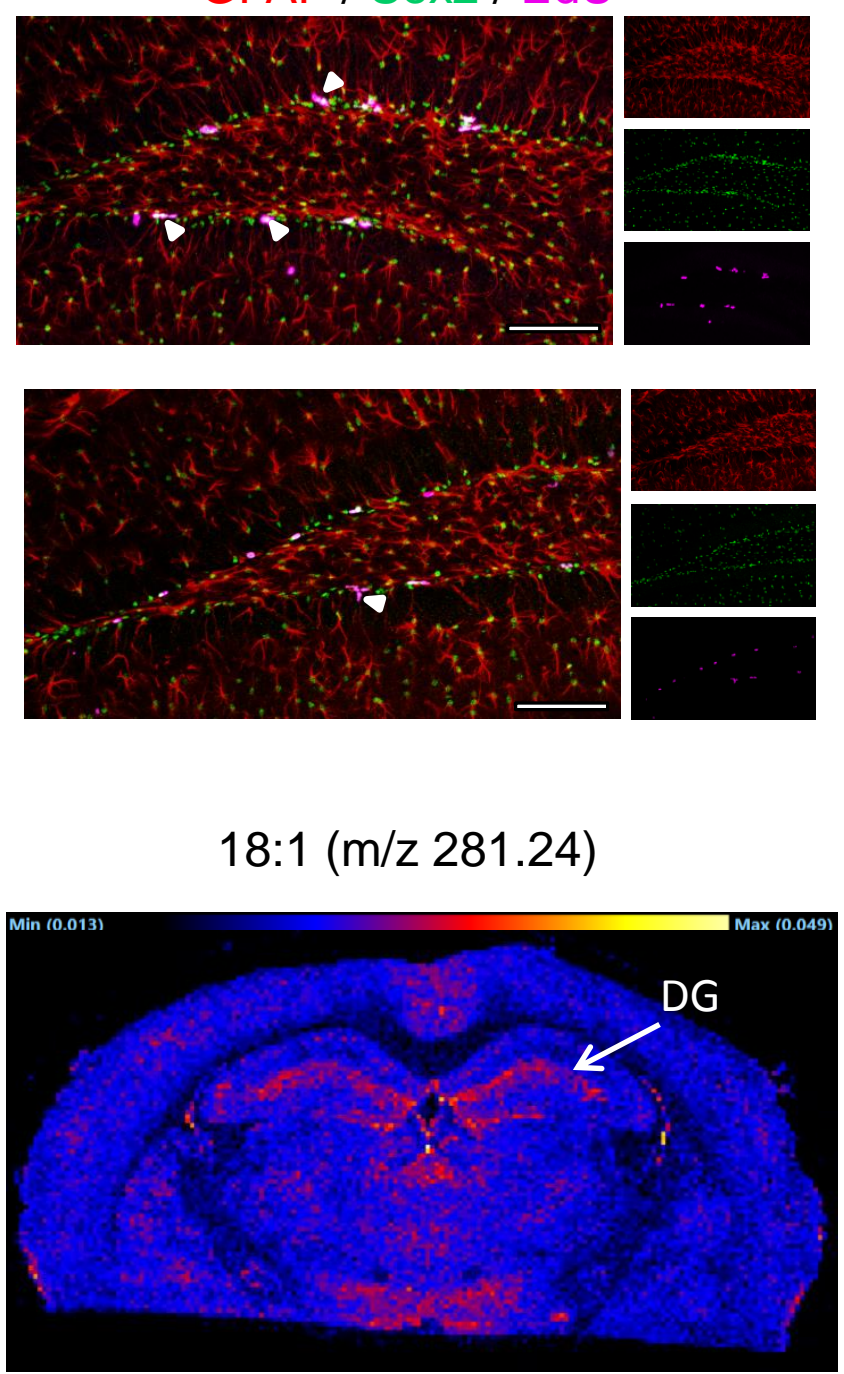
A

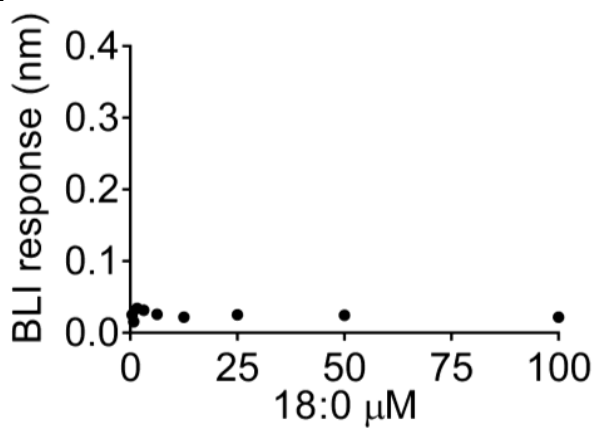

B

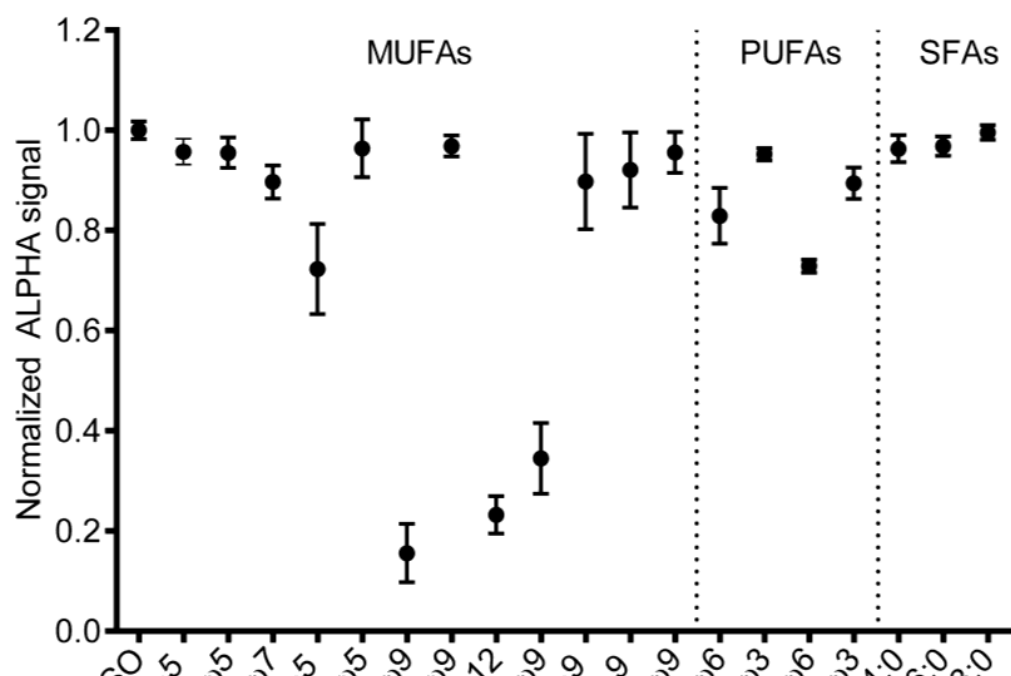

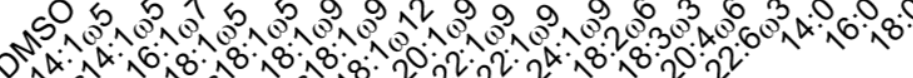

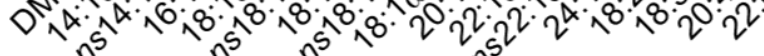

D

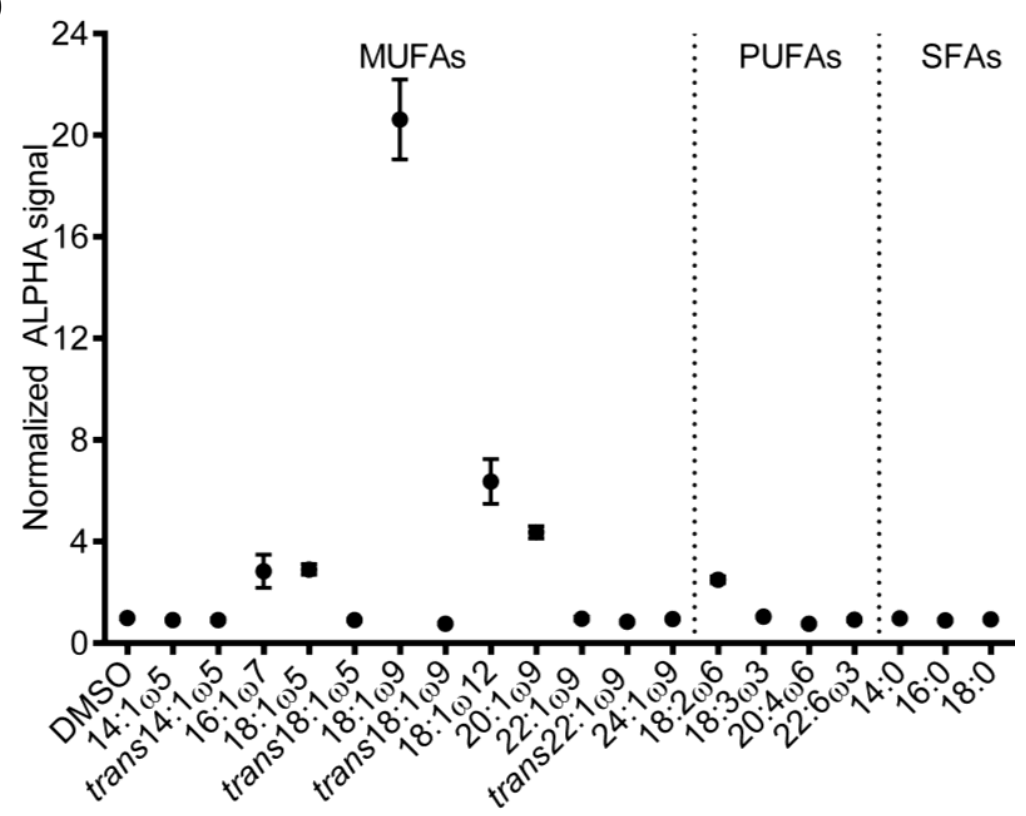

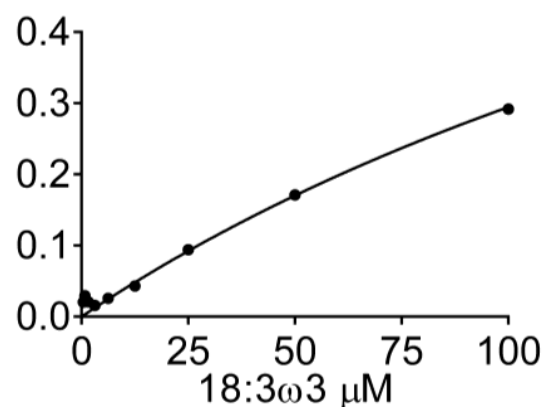

C

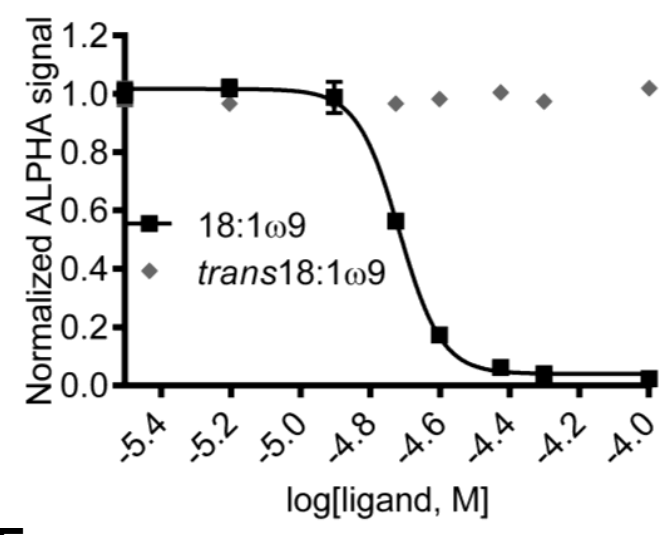

E

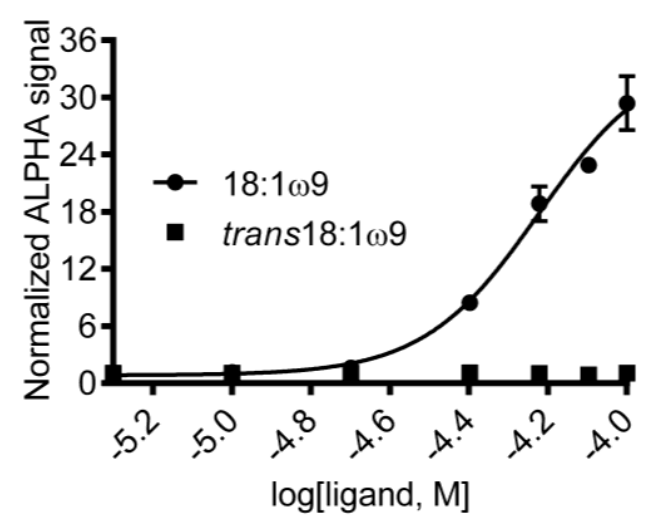

$\mathbf{F}$

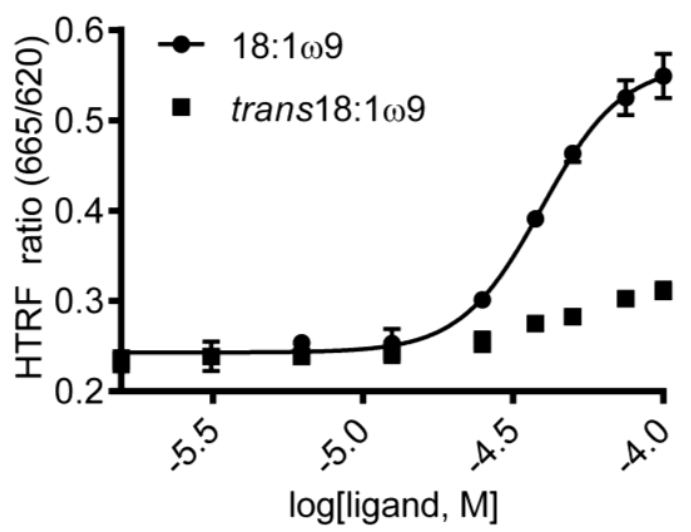

Figure 2 
A

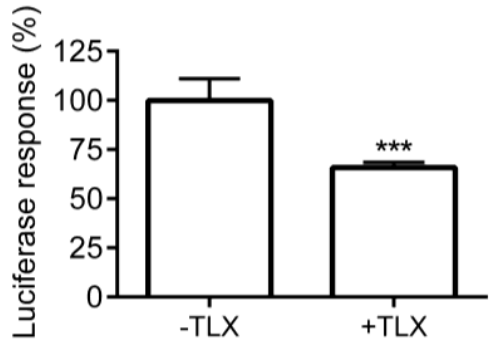

B

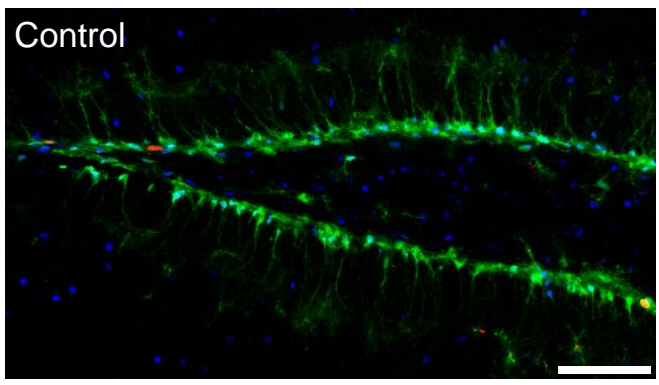

C

Sham, 18:3w3, or $18: 1 \omega 9$ AP $-1.7,-2.1,-2.5$

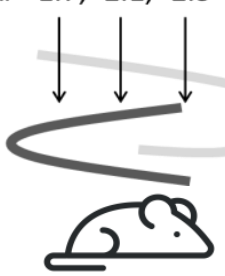

Lfng-eGFP
E

TMX Inj EdU SAC Ohr $48 \mathrm{hr} \quad 54 \mathrm{hr} \quad 70 \mathrm{hr} \quad 72 \mathrm{hr}$

Sham or $18: 1 \omega 9$ AP -1.7, -2.1, -2.5

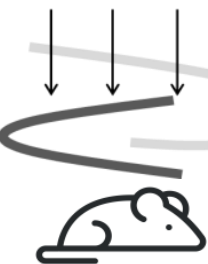

iTIX

$\mathrm{i} T / x^{+/+}$

Figure 3

\section{Lfng-eGFP / EdU / Sox2}
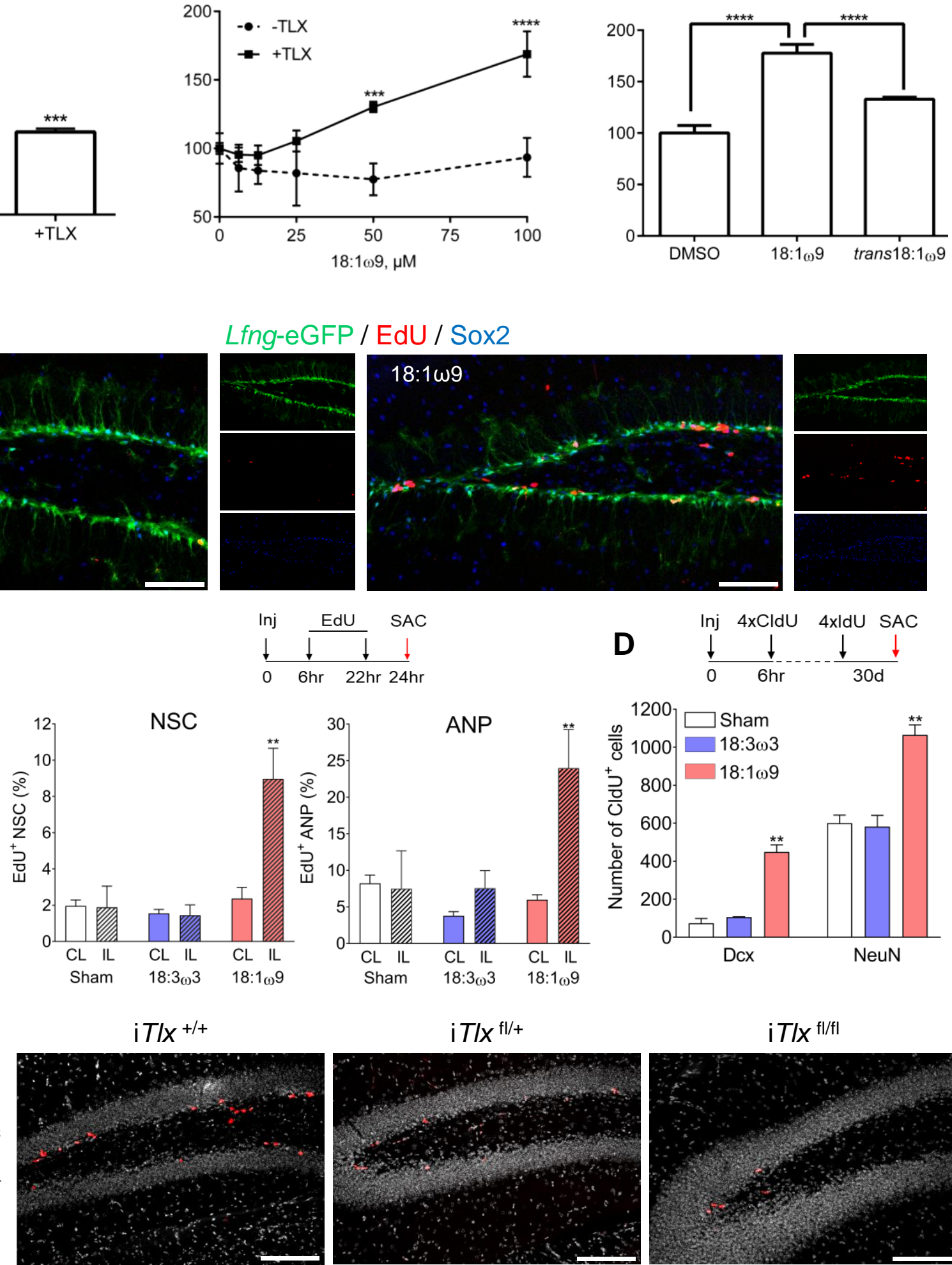

$\mathrm{i} T / x^{\mathrm{fl} /+}$

$\mathrm{i} T / x^{\mathrm{fl} / f \mathrm{l}}$
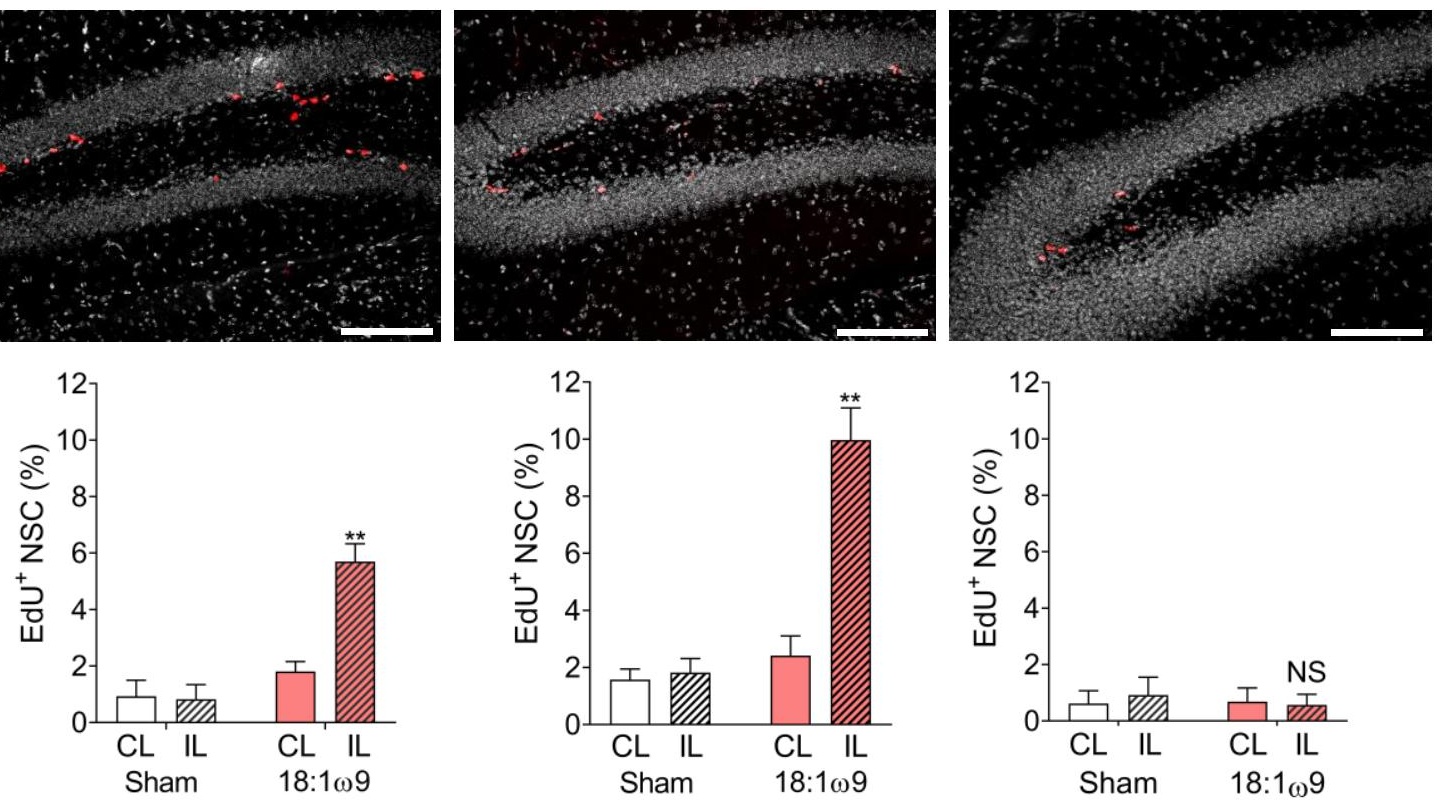
A

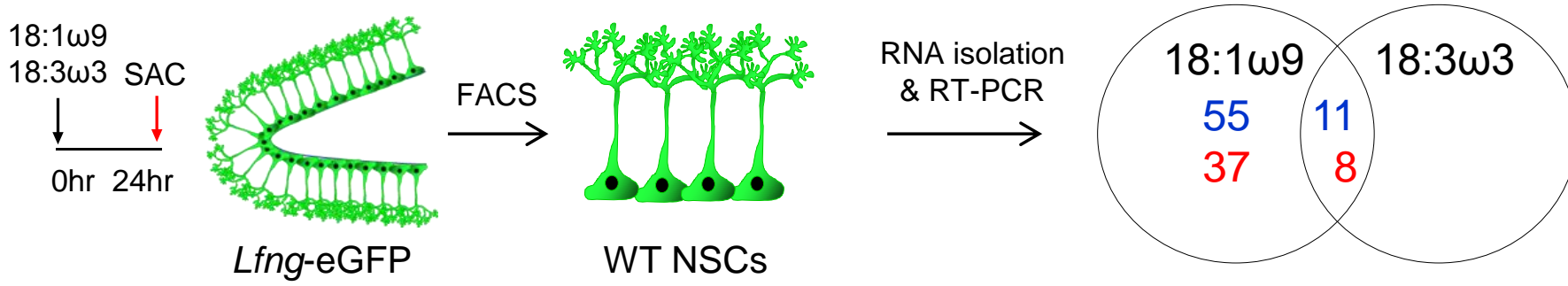

B

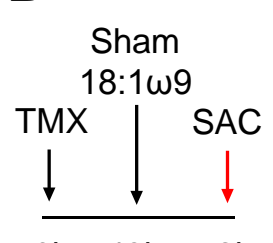

Ohr 48hr $72 \mathrm{hr}$

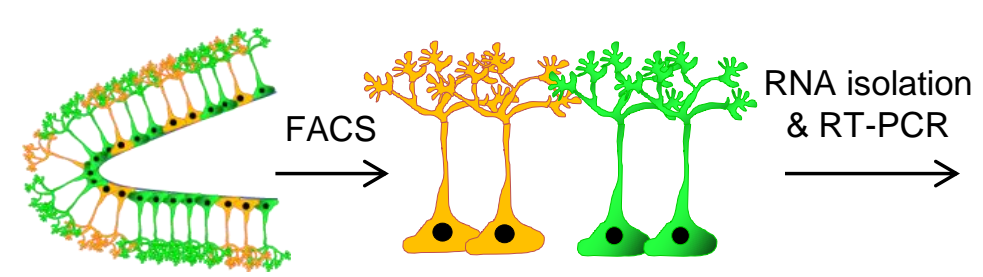

$\mathrm{i} T / x^{\mathrm{fl} / \mathrm{fl}}$

i $T / x^{f \mid / f l}$ NSCs $T / x^{+/+}$NSCs

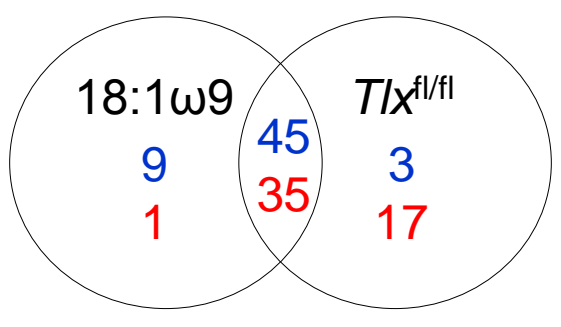

Cell Cycle

\begin{tabular}{|r|c|c|}
\hline Mki67- & 9.88 & 6.00 \\
Itgb1- & 10.52 & 4.85 \\
Stag1- & 11.69 & 5.28 \\
Pkd1- & 12.65 & 6.26 \\
\hline Gpr132- & 10.58 & 4.64 \\
\hline Msh2- & 14.39 & 9.71 \\
Rbl2- & 11.16 & 6.48 \\
\hline Tfdp1- & 9.23 & 4.88 \\
Hus1- & 10.59 & 3.19 \\
Cdk2- & 12.44 & 8.74 \\
Ran- & 8.76 & 6.65 \\
\hline Terf1- & 12.43 & 9.75 \\
E2f2- & 9.36 & 8.14 \\
Cdc6- & 7.64 & 8.66 \\
Rad17- & 10.12 & 9.99 \\
Chek2- & 9.37 & 11.92 \\
\hline
\end{tabular}

18:1w9 Sham $i T L X^{\text {IIIII }}$ vs. vs.

Sham WT Sham T/X+/+

\begin{tabular}{|r|c|c|}
\cline { 1 - 1 } Abl1- & 7.93 & 10.13 \\
Aurka- & 4.36 & 8.38 \\
Sfn- & 4.87 & 4.32 \\
E2f3- & 4.34 & 3.66 \\
Ccnd1- & 4.54 & 3.20 \\
Casp3- & 5.42 & 5.39 \\
Ccnc- & 3.93 & 5.75 \\
Trp63- & 4.65 & 6.44 \\
Cdk1- & 5.10 & 6.51 \\
Atr- & 4.31 & 5.84 \\
Bcl2- & 5.84 & 7.64 \\
Brca1- & 5.73 & 6.46 \\
Cdk5rap1- & 7.05 & 6.98 \\
Rbl1- & 3.32 & 6.54 \\
Mcm2- & 4.42 & 6.94 \\
Trp53- & 2.53 & 4.43 \\
\hline
\end{tabular}

18:1 w9 Sham $i T L X^{1 / 1 / 1}$ vs. vs.

Sham WT Sham T/X+/+

\begin{tabular}{r|cc|}
\cline { 2 - 3 } Gpi1- & 4.80 & 2.21 \\
Nrcam- & 5.38 & 3.82 \\
Mtap2- & 4.73 & 3.49 \\
Fgf2- & 5.75 & 5.23 \\
Hdac4- & 3.35 & 3.77 \\
Rtn4- & 8.02 & 7.09 \\
Nf1- & 7.33 & 10.61 \\
Pou3f3- & 6.52 & 11.29 \\
Erbb2- & 3.38 & 9.46 \\
Dvl3- & 3.95 & 8.66 \\
Ndn- & 3.05 & 8.69 \\
Notch1- & 4.27 & 6.71 \\
Ascl1- & 4.85 & 8.30 \\
Drd2- & 4.56 & 7.70 \\
Pard3- & 4.40 & 6.77 \\
Th- & 3.76 & 6.09 \\
\hline & $18: 1 \omega 9$ & Sham iTL \\
vs. & vs. \\
Sham WT & Sham $T / X^{+/+}$
\end{tabular}

Neurogenesis

\begin{tabular}{|c|c|c|}
\hline Alk- & 3.50 & 6.93 \\
\hline $\mathrm{Nr} 2 \mathrm{e} 3-$ & 2.99 & 7.78 \\
\hline Mef2c- & 3.20 & 7.30 \\
\hline DII1- & 3.31 & 6.12 \\
\hline MII1- & 4.26 & 7.99 \\
\hline Nrp2- & 3.07 & 5.97 \\
\hline Cdk5r1- & 2.39 & 5.14 \\
\hline II3 & 2.05 & 11.08 \\
\hline Neurog1 & 2.18 & 6.86 \\
\hline Shh- & 2.29 & 6.80 \\
\hline Hey1- & 3.46 & 4.57 \\
\hline Artn- & 2.93 & 4.92 \\
\hline Heyl- & 5.10 & 6.79 \\
\hline Pax6 & 4.98 & 6.53 \\
\hline Tnr & 5.44 & 8.34 \\
\hline Nrp1- & 4.73 & 8.73 \\
\hline & $\begin{array}{l}\text { 18:1w9 } \\
\text { vs. }\end{array}$ & $\begin{array}{c}\text { Sham i } T L X^{1 / 1 / 1} \\
\text { vs. } \\
\text { Sham } T / X^{+/+}\end{array}$ \\
\hline
\end{tabular}

